\title{
Laser resurfacing of the aging face
}

\author{
Jason N. Pozner ${ }^{1,2}$, Barry E. DiBernardo ${ }^{3}$, Jonathan Cook ${ }^{1}$ \\ 'Sanctuary Plastic Surgery, Boca Raton, FL 33431, USA. \\ ${ }^{2}$ Cleveland Clinic Florida, Weston, FL 33331, USA. \\ ${ }^{3}$ New Jersey Plastic Surgery, Montclair, NJ 07042, USA
}

Correspondence to: Dr. Jason Pozner, Sanctuary Plastic Surgery, 4800 N Federal Hwy c101, Boca Raton, FL 33431, USA. E-mail: jpoznermd@gmail.com

How to cite this article: Pozner JN, DiBernardo BE, Cook J. Laser resurfacing of the aging face. Plast Aesthet Res 2021;8:20. https://dx.doi.org/10.20517/2347-9264.2020.218

Received: 15 Dec 2020 First Decision: 22 Feb 2021 Revised: 6 Mar 2021 Accepted: 23 Mar 2021 Available online: 8 Apr 2021

Academic Editor: Raúl González-García Copy Editor: Yue-Yue Zhang Production Editor: Yue-Yue Zhang

\begin{abstract}
Laser technology has evolved significantly over the last 30 years, and laser devices have become integral tools for skin rejuvenation in the hands of many plastic surgeons practicing today. The purpose of this article is to briefly review the history of aesthetic laser technology, to discuss patient selection and expectations for various laser devices, and finally to review the technology and applications of these devices.
\end{abstract}

Keywords: Lasers, plastic surgery, facial rejuvenation, laser resurfacing, aesthetic surgery, laser devices

\section{INTRODUCTION}

Following the introduction of the carbon dioxide $\left(\mathrm{CO}_{2}\right)$ laser for skin resurfacing in the mid-1990s, the technology achieved rapid popularity and even replaced chemical peels and dermabrasion in many practices. The $\mathrm{CO}_{2}$ laser has a wavelength of $10,600 \mathrm{~nm}$, an absorbing chromophore of water, and is used to vaporize tissue [Figure 1A]. Continuous mode lasers were initially used, but complications were encountered due to excessive depths of ablation and thermal damage. This led to the development of short pulse laser devices in an effort to minimize complications. Short pulse lasers created a tissue exposure time of less than one millisecond, which allowed tissue ablation with limited residual thermal damage of approximately 75-100 $\mu \mathrm{m}$. Short-term results of eradicating wrinkles and tightening lax tissue were excellent, but longer-term follow-up showed hypopigmentation in a large percentage of patients. These pigmentary complications and the considerable downtime created for the patients led to the demise of "full field" $\mathrm{CO}_{2}$ laser resurfacing around the turn of the century ${ }^{[1]}$. 
A

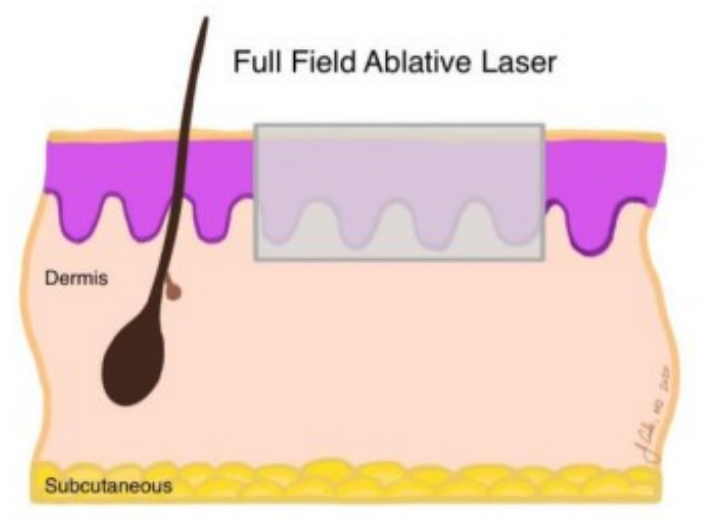

B

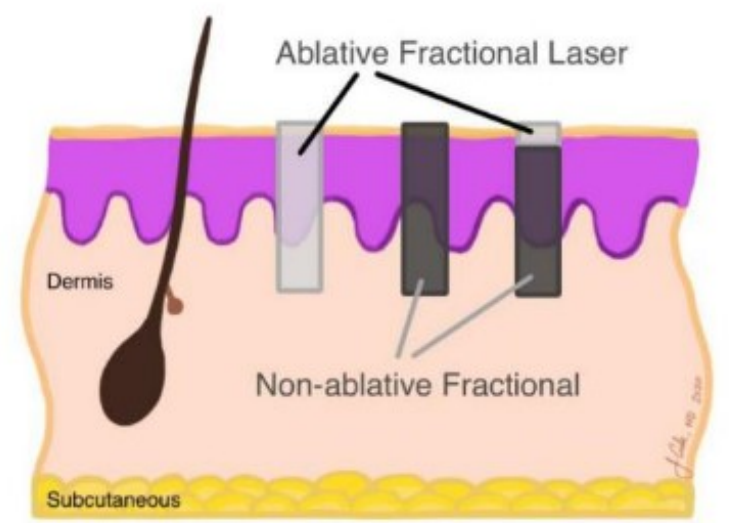

Figure 1. Skin graphic.

Erbium:YAG lasers (“Er:YAG”) (2940 nm) were introduced around 2000 and marketed for superficial resurfacing. Erbium lasers have a higher water absorption coefficient than $\mathrm{CO}_{2}$ lasers and ablate tissue with much less thermal damage $(5-10 \mu \mathrm{m})^{[2]}$. Initial machines were low powered, lacked pattern generators, and needed considerable quantities of passes and treatment time to achieve deeper depths of ablation. Subsequent systems had more significant power and could be used for efficient deeper resurfacing. There is a linear relationship between the energy delivered and depth of ablation, with approximately $3-4 \mu \mathrm{m}$ ablated per joule of Er:YAG laser fluence delivered. Complications were fewer, yet downtime appeared to be similar to that of $\mathrm{CO}_{2}$ systems. Conclusions of comparative studies were that the combined depth of ablation and coagulation were the determining factors in length of recovery ${ }^{[3,4]}$. Combination systems of $\mathrm{CO}_{2}$ and Er:YAG lasers were popular for a short time (Derma-K", Lumenis lasers, Yokneam, Israel) with the beams being delivered either sequentially or simultaneously.

Variable or long-pulse Er:YAG lasers (Sciton Inc, Palo Alto, CA) allow control over the amount of residual thermal injury produced for a given amount of tissue removal. These variable pulse Er:YAG systems seem to produce skin tightening and wrinkle reduction similar to $\mathrm{CO}_{2}$ lasers with a much shorter period of erythema and a much lower risk of hypopigmentation. These devices remain very popular today.

In 2004, Manstein et al ${ }^{[5]}$ introduced the concept of fractional photothermolysis and it transformed the field of laser resurfacing. Whereas full field laser resurfacing (described above) removes the entire skin surface in the treatment area, with the depth of injury determined by energy level, fractional laser resurfacing treats only a small "fraction" of the skin at each session, leaving skip areas between each treatment zone [Figure 1B].

Fractional laser resurfacing was first performed commercially using non-ablative fluences at $1550 \mathrm{~nm}$ (Solta Medical, Mountain View, CA). These non-ablative fractional lasers created a column of thermal damage with intact epidermis. Healing occurred from deeper structures as well as from adjacent structures. This differs from full field resurfacing in which healing occurred from only deeper structures. Deeper treatments (i.e., to the reticular dermis) can more safely be performed using this approach than would be tolerated using a full field treatment. Advantages of this approach include avoidance of an open wound and very low risk of pigment disturbance or scarring. Disadvantages include the need for multiple treatments, and a somewhat limited clinical response when compared to full field ablative resurfacing ${ }^{[6-13]}$. Since the 
introduction of the original system there have been many manufacturers that have introduced similar nonablative fractional devices with wavelengths of 1440, 1540, and $1550 \mathrm{~nm}$. These devices differ in power output, spot size, density, etc., and comparisons of clinical efficacy are difficult, yet similar degrees of tissue injury should produce similar clinical results.

Fractional ablative resurfacing with $\mathrm{CO}_{2}$, erbium, and yttrium-scandium-gallium-garnet (YSGG) systems was introduced with the intent of providing more significant results than non-ablative fractional systems while achieving shorter healing times and complications when compared with full field ablative systems [ Figure 1$]^{[14]}$. These devices differ not only in wavelength but in system power, spot size, and the amount of thermal damage created adjacent to and deep to the ablated area. One popular erbium system, the Sciton ProFractional, allows one to vary the amount of thermal damage similarly to their full field system. Other newer $\mathrm{CO}_{2}$ fractional lasers allow variation of the thermal damage zones (Deka Medical, Calenzano, Italy) while others allow superficial and deeper penetration with a single scan (Syneron, Yokneum, Israel). As with non-ablative fractional systems, direct comparison between devices is difficult as devices differ in power output, spot size, density, and degree of thermal damage, but similar degrees of injury should produce similar clinical results.

One of the newest fractional lasers on the market is a hybrid fractional laser made by Sciton (Palo Alto, CA) and called the Halo. This is a very interesting device which allows coincident delivery of their erbium fractional laser followed by a non-ablative $1470 \mathrm{~nm}$ pulse in the same treatment zone [Figure $1 \mathrm{~B}$, far right $]^{[15]}$. This device is very efficacious and creates very minimal healing times. This is the authors' laser choice for skin rejuvenation when recovery time is limited.

The newest wavelength to be introduced into the fractional arena is $1927 \mathrm{~nm}$ (with devices offered by Solta Medical, Lutronic, and Sciton). These non-ablative fractional devices are especially effective in removing superficial pigment.

Full field ablative resurfacing and both fractional ablative and non-ablative systems remain very popular in clinical use at this time.

\section{PATIENT SELECTION}

Careful patient selection and a clear understanding of potential complications are important to achieving consistent results. The most common indications for both full field and fractional laser resurfacing are superficial dyschromias, dermatoheliosis, textural anomalies, superficial to deep rhytids, acne scars, and surgical scars. Other conditions that may respond favorably include rhinophyma, sebaceous hyperplasia, xanthelasma, syringomas, actinic cheilitis, and diffuse actinic keratoses. Dyschromias such as melasma have been successfully treated with fractional resurfacing but results are not consistent. The usual area for resurfacing is the face, but body and neck skin may be treated with variations of technique. Non-facial areas lack the appendages necessary for skin rejuvenation and treatment must be performed non-aggressively to avoid complications. These devices are generally used with patients with Fitzpatrick skin types I-IV, but can be used in skin types V and VI with modification of technique to avoid overdelivery of thermal energy.

Patient assessment begins at the initial consultation with observation of the patient's Fitzpatrick skin type, ethnicity, and pathology to be treated. For example, deep acne scarring will not be successfully treated with a single treatment of non-ablative fractional treatment, but mild textural issues may respond to superficial treatment. The next assessment is of the patient's tolerance of healing period "downtime". A busy executive with no urgency for clinical results may be able to be treated only with a series of no-downtime non-ablative 
fractional treatments, whereas the bride's mother looking for maximum improvement in a short time to look her best for her daughter's wedding may need a single session with more aggressive treatment. The last parameter is one not usually discussed in medical journals or book chapters: patient finances. A deep full field resurfacing performed under general anesthesia will be more expensive for the patient than a superficial treatment performed with topical anesthesia. However, in patients with deep rhytids a more aggressive procedure under general anesthesia may be more cost effective than multiple superficial treatments. Another consideration is laser resurfacing while patients are undergoing other procedures such as facelift, abdominoplasty, or aesthetic breast surgery. These patients often have "built-in downtime" from other procedures, and therefore have the recovery time needed for deep resurfacing.

Many of us with various devices in our offices can offer patients a plethora of treatment options and this can be very confusing to the patients. An effective consultation will encompass a thorough evaluation of the pathology and provide options to the patients in terms of downtime, efficacy, risks, and cost.

\section{Expected benefits and alternatives}

The potential for improvement depends upon the device used and depth and degree of injury produced. There are many options for superficial treatment of texture issues, dyschromias, and superficial rhytids including non-aggressive full field resurfacing with Er:YAG, $\mathrm{CO}_{2}$, YSGG, or with non-ablative or ablative fractional treatment. Many practitioners are using combination therapy with superficial full field treatment combined with fractional treatment, whereas others are combining fractional ablative and non-ablative therapy and others again are using intense pulsed light (IPL) therapy combined with resurfacing. Other treatments that may yield similar results for superficial pathologies include light chemical peels such as $15 \%$ $30 \%$ trichloroacetic acid, IPL devices, and Q-switched lasers (532 nm for dyschromias). We prefer lasers to chemical peels owing to the uniformity and predictability of treatment as the device produces tissue effects with minimal variability from pulse to pulse or patient to patient. The learning curve with lasers is less than with chemical peels due to the predictability of the treatment. Expert chemical peelers may get similar results to laser treatment at a fraction of the laser cost, but years of experience are necessary to achieve consistency of results. IPL devices may be used to treat dyschromias and superficial vasculature, but require multiple sessions and do not address textural issues or rhytids. Q-switched lasers (532, 694, and $755 \mathrm{~nm}$ ) are excellent at removing dyschromias in one session but have resultant erythema that may last for up to 10 days.

More significant pathology requires deeper treatment to achieve results in a single session. There is still a question of whether repeated superficial therapies with ablative fractional devices will achieve similar results to one more aggressive full field session. Deep ablative full field resurfacing may be performed with either erbium or $\mathrm{CO}_{2}$ systems. YSGG in full field mode does not ablate deep enough to treat more significant pathology. Acne scars appear to respond better to fractional therapy than to full field therapy. Alternative treatments may be deeper chemical peels such as phenol or dermabrasion. The authors feel that lasers provide more consistent and reproducible results than chemical peels or dermabrasion.

\section{LASERS AND TECHNICAL OVERVIEW}

As discussed above, current devices used for ablative laser resurfacing include $\mathrm{CO}_{2}$, Er:YAG, and YSGG lasers, in both full field and fractional modes, and non-ablative devices in a variety of wavelengths including 1440, 1540, 1550, and $1927 \mathrm{~nm}$. Some machines offer upgradeable expandable platforms where full field devices and fractional devices are available in one machine, whereas other companies offer only isolated full field or fractional devices. 


\section{$\mathrm{CO}_{2}$ full field}

Pulsed or scanned full field $\mathrm{CO}_{2}$ lasers were very popular from 1995 to about 2000. These devices were powerful with a typical single pass ablating approximately $75 \mu \mathrm{m}$ and creating thermal damage of approximately 75-100 $\mu \mathrm{m}$. This residual area of desiccated tissue reduced the amount of absorbing chromophore (water) and made subsequent passes less efficient, and in fact excessive stacked passes acted as a heat sink and created excessive thermal damage and the potential for scar. Up to three passes were usually performed with the original $\mathrm{CO}_{2}$ resurfacing lasers owing to diminishing efficiency of tissue removal and rapidly increasing risk of complications. The ablated tissue and underlying thermal damage led to long-term collagen changes and tissue remodeling. Healing time with deep full field $\mathrm{CO}_{2}$ laser resurfacing took approximately 10-14 days and caused erythema that typically lasted a few months. Complications of prolonged erythema and delayed hypopigmentation occurred and led to diminished use of these devices.

\section{Er:YAG full field}

The erbium:YAG laser $\left(2940 \mathrm{~nm}\right.$ ) has an absorption coefficient 10 times greater than the $\mathrm{CO}_{2}$ laser, which ablates tissue more efficiently and leaves less residual thermal damage $(5-10 \mu \mathrm{m})$. There is a linear relationship between energy density (fluence) delivered and tissue ablated with 3-4 $\mu \mathrm{m}$ of tissue removed per $\mathrm{J} / \mathrm{cm}^{2}$ and multiple passes can be used to produce deeper tissue removal without additive residual thermal injury. This leads to recovery time of deep full field Er:YAG laser resurfacing of 7-10 days to full epithelialization followed by 3-6 weeks of erythema. Superficial and deep resurfacing can be performed with these devices with increasing results and increasing recovery times with deeper treatments [Figure 2 and Figure 3]. Complications including hypopigmentation occur less often than with $\mathrm{CO}_{2}$ laser full field resurfacing.

Variable pulse Er:YAG systems allow a shorter ablative pulse followed by longer subablative pulses to create increasing thermal damage. These devices are typically used to achieve $\mathrm{CO}_{2}$ laser like results, but without the long healing times and complications such as hypopigmentation.

\section{YSGG full field}

The $2790 \mathrm{~nm}$ yttrium-scandium-gallium-garnet (Pearl: Cutera, Brisbane, California) provides half the affinity for water as the Er:YAG laser at 2940. This device in full field mode causes ablation of approximately 20-30 $\mu \mathrm{m}$ and residual thermal damage of approximately $20 \mu \mathrm{m}$ per pass. Healing times and downtime are a few days. Deeper resurfacing is not performed with this device.

\section{Non-ablative fractional technology}

As previously mentioned, non-ablative fractional resurfacing involves the simultaneous or sequential placement of multiple small spots of laser light onto the surface of the skin with intervening skip areas of unexposed skin. The chromophore used is water and the wavelengths used are 1440, 1470, 1550, and 1540 $\mathrm{nm}$. The lasers create a column of tissue coagulation from 300 to $1200 \mu \mathrm{m}$ and are called microthermal zones. The newest non-ablative fractional wavelength is $1927 \mathrm{~nm}$ (Thulium) and downtime appears to be less then with other non-ablative fractional lasers.

\section{Fractional ablative technology}

Ablative fractional resurfacing can be performed with $\mathrm{CO}_{2}$, Er:YAG, and YSGG devices. There are many devices available from many well-known laser manufacturers. Differences in devices are the mode of spot placement - scanning versus stamping, size of holes (width and depth) created, and power output of devices. Differences between fractional $\mathrm{CO}_{2}$ systems, fractional Er:YAG systems, and YSGG systems are similar to their full field counterparts in that the $\mathrm{CO}_{2}$ systems cause leave more residual thermal damage. Newer Er:YAG systems have variable pulse widths, which cause carbon-dioxide-like thermal damage. Re- 


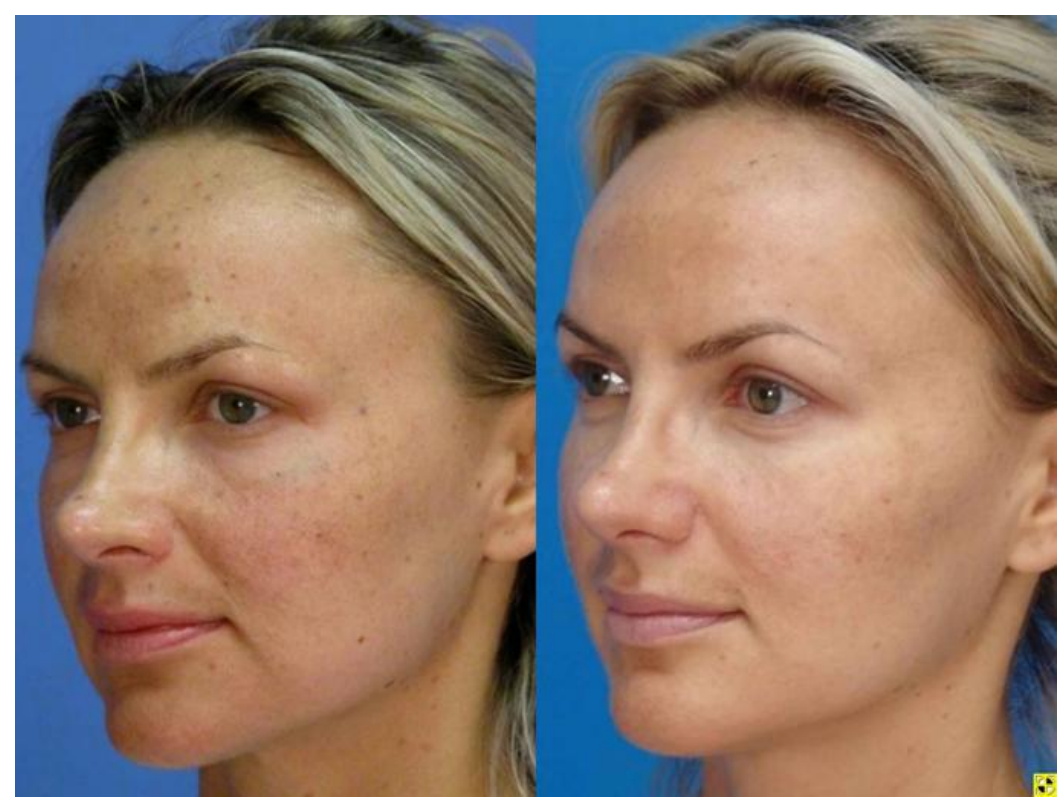

Figure 2. Full field resurfacing 1 (Superficial).

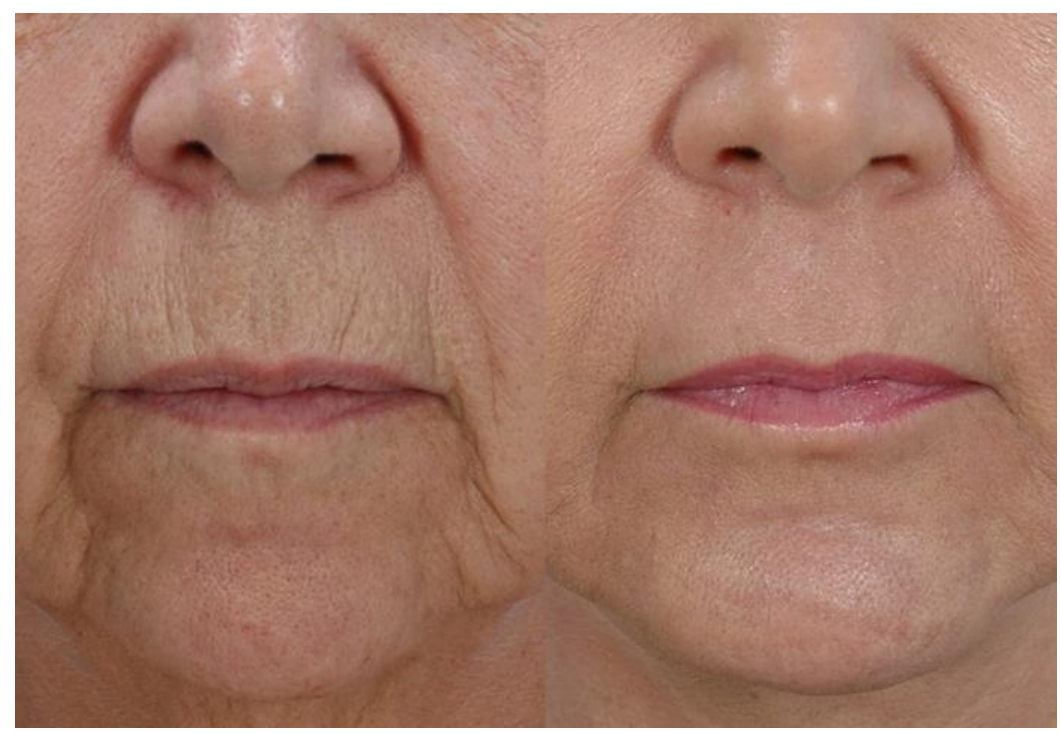

Figure 3. Full field resurfacing 2 (Deep).

epithelialization is quicker than with full field ablation and recovery time varies from hours to a few days depending upon depth and density of treatment.

Both ablative fractional and non-ablative fractional devices are used to treat acne and other scars [Figure 4]. Multiple treatments are needed and there is no current consensus as to the best technology for this at present. It was very common in our offices to perform combination treatment with superficial full field Er:YAG resurfacing followed by Er:YAG fractional treatment. The superficial Er:YAG treatment improves skin texture and minor irregularities while the fractional treatment is useful for collagen remodeling. This treatment regimen was replaced by treatment with a hybrid fractional laser with or without simultaneous 

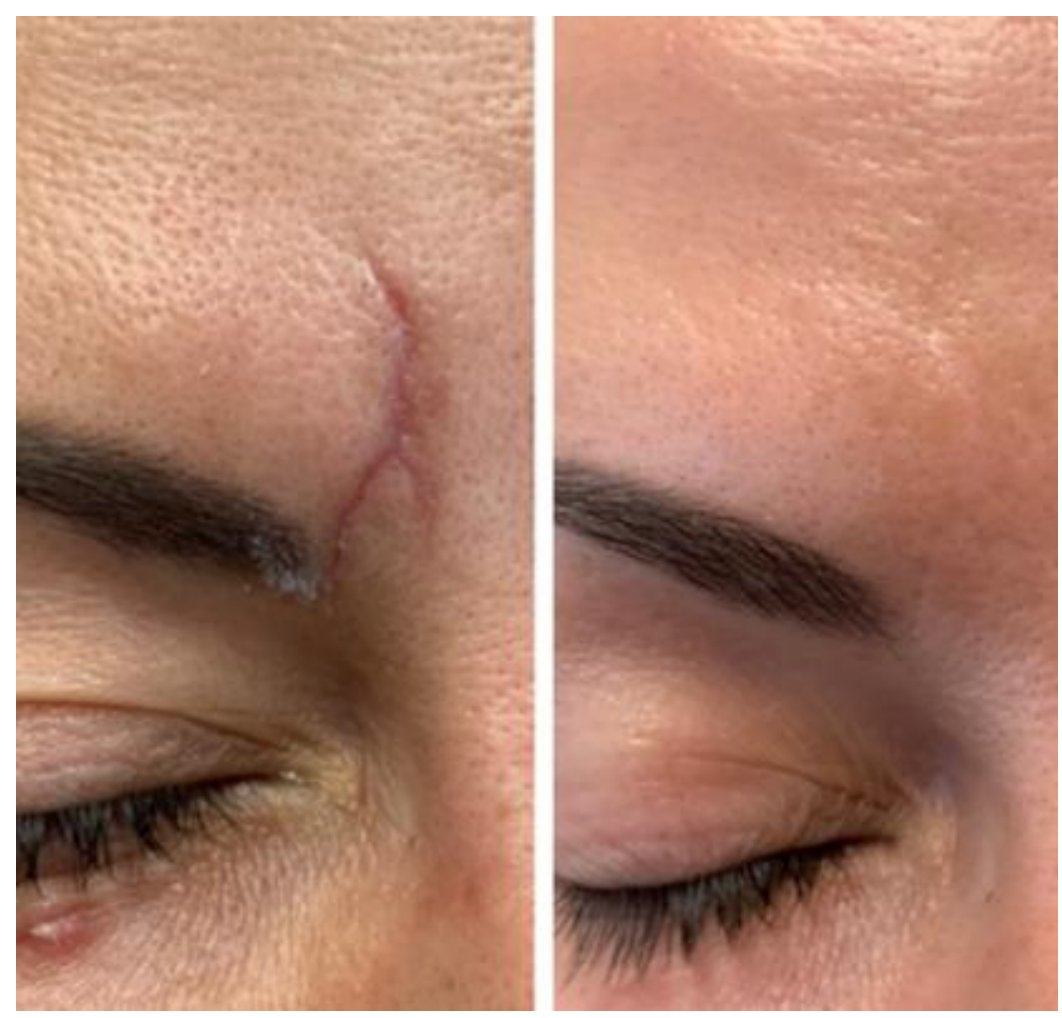

Figure 4. Laser scar.

IPL treatment.

As fractional $\mathrm{CO}_{2}$ treatments have been pushed to higher and higher coverages in an attempt to maximize efficacy, healing times predictably have increased. More importantly, complications such as scarring and hypopigmentation have been observed at coverages in excess of $45 \% . \mathrm{CO}_{2}$ resurfacing histology consistently shows a significant component of tissue ablation and coagulation. Efficacious resurfacing is believed to require a significant component of both. One strategy that has been explored to increase coverage percentage and maximize efficacy involves a combination treatment with ablative Er:YAG fractional and non-ablative fractional exposures in a single treatment session. This provides a component of largely ablative exposure with the fractional Er:YAG treatment and a component of coagulation with the nonablative fractional treatment. Rather than being spatially overlapped as in a fractional $\mathrm{CO}_{2}$ microthermal zone, the coagulation and ablation are separated. Coverages up to $65 \%$ are routinely applied with only a modest increase in healing time and erythema compared with fractional Er:YAG treatment alone and somewhat less than that reported for fractional $\mathrm{CO}_{2}$. Advantages of this approach include preservation of the short recovery and low incidence of complications seen with fractional Er:YAG treatments and the potential for significant improvement even in perioral rhytids. Disadvantages include the need for two lasers or a single laser platform that offers both options and the time-consuming nature of the treatments.

This combined treatment regimen led to the introduction of the Sciton Halo hybrid laser. This device allows either non-ablative fractional resurfacing with a $1470 \mathrm{~nm}$ laser or coincident treatment with both a fractional ablative wavelength $(2940 \mathrm{~nm})$ and a fractional non-ablative wavelength $(1470 \mathrm{~nm})$. Treatment with this device has very little downtime, and the results of coincident ablative/non-ablative resurfacing on pigment, texture and pores appear to surpass that achieved with either non-ablative or ablative fractional resurfacing 


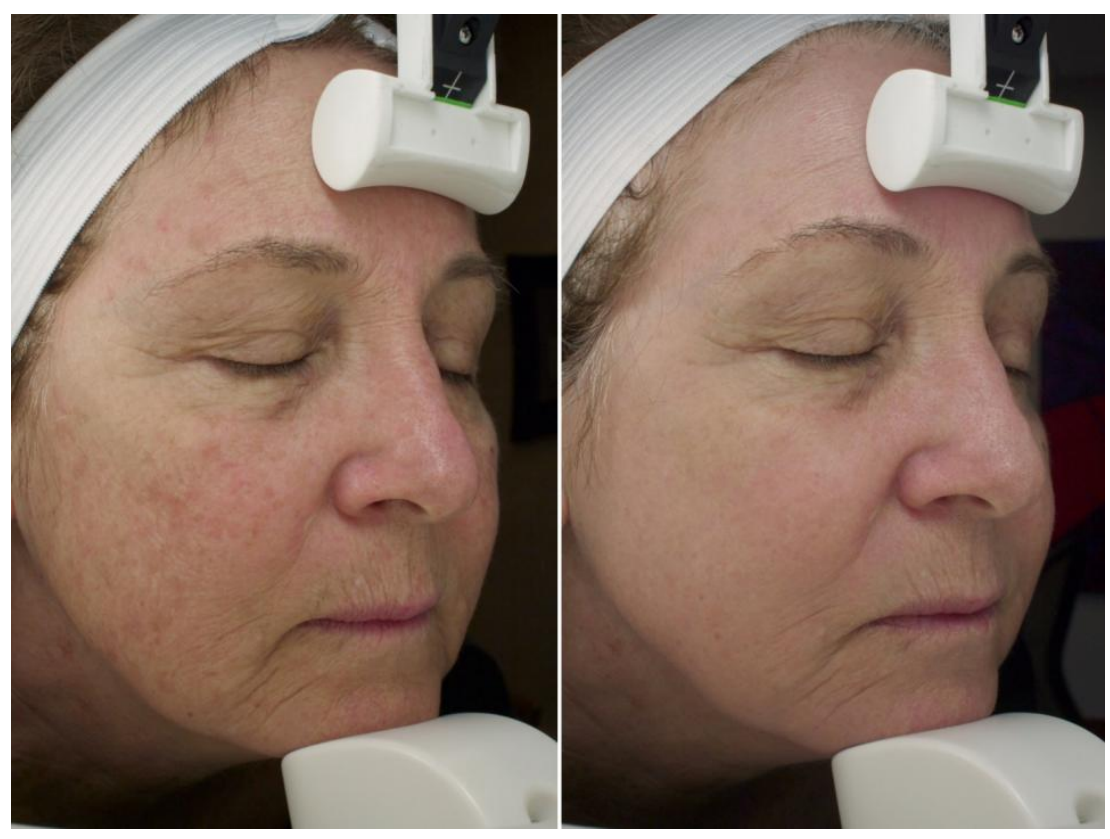

Figure 5. Halo.

alone [Figure 5]. Fewer treatments are needed then with other fractional devices to achieve similar results. Newer protocols with this device are for combined treatment with an IPL device and results on pigment are better than non-combined treatment.

In summary, excellent results in laser resurfacing are achieved through appropriate patient selection, realistic expectations (on the part of the physician and patient), and a keen understanding of the various technologies available.

\section{DECLARATIONS}

\section{Authors' contributions}

To the design of this review, to the writing of this manuscript, and to the editorial revisions during submission to this journal: Pozner JN, DiBernardo BE, Cook J

\section{Availability of data and materials}

Not applicable.

\section{Financial support and sponsorship}

No financial support was received for this article.

\section{Conflicts of interest}

All authors declared that there are no conflicts of interest.

\section{Ethical approval and consent to participate}

Not applicable.

\section{Consent for publication}

Written consent was obtained for publication by all patients. 


\section{Copyright}

(c) The Author(s) 2021.

\section{REFERENCES}

1. Weinstein C, Pozner J, Ramirez O. Complications of carbon dioxide laser resurfacing and their prevention. Aesthet Surg J 1997;17:216-25. DOI PubMed

2. Bass LS. Erbium:YAG laser skin resurfacing: preliminary clinical evaluation. Ann Plast Surg 1998;40:328-34. DOI PubMed

3. Pozner JM, Goldberg DJ. Histologic effect of a variable pulsed Er:YAG laser. Dermatol Surg 2000;26:733-6. DOI PubMed

4. Pozner JN, Roberts TL. Variable-pulse width Er:-YAG laser resurfacing. Clin Plast Surg 2000;27:263-71. PubMed

5. Manstein D, Herron GS, Sink RK, Tanner H, Anderson RR. Fractional photothermolysis: a new concept for cutaneous remodeling using microscopic patterns of thermal injury. Lasers Surg Med 2004;34:426-38. DOI PubMed

6. Geronemus RG. Fractional photothermolysis: current and future applications. Lasers Surg Med 2006;38:169-76. DOI PubMed

7. Laubach HJ, Tannous Z, Anderson RR, Manstein D. Skin responses to fractional photothermolysis. Lasers Surg Med 2006;38:142-9. DOI PubMed

8. Laubach HJ, Tannous Z, Anderson RR, Manstein D. A histological evaluation of the dermal effects after fractional photothermolysis treatment. Lasers Surg Med 2005;36:86.

9. Rahman Z, Rokhsar CK, Tse Y, Fitzpatrick R. The treatment of photodamage and facial rhytides with fractional photothermolysis. Lasers Surg Med 2005;36:32.

10. Rahman Z, Alam M, Dover JS. Fractional laser treatment for pigmentation and texture improvement. Skin Therapy Lett 2006;11:7-11. PubMed

11. Tannous Z, Laubach HJ, Anderson RR, Manstein D. Changes of epidermal pigment distribution after fractional resurfacing: a clinicopathologic correlation. Lasers Surg Med 2005;36:26-32.

12. Wanner M, Tanzi EL, Alster TS. Fractional photothermolysis: treatment of facial and nonfacial cutaneous photodamage with a 1,550nm erbium-doped fiber laser. Dermatol Surg 2007;33:23-8. DOI PubMed

13. Weiss RA, Gold M, Bene N, et al. Prospective clinical evaluation of 1440-nm laser delivered by microarray for the treatment of photoaging and scars. J Drugs Dermatol 2006;5:740-4. PubMed

14. Clementoni MT, Gilardino P, Muti GF, Beretta D, Schianchi R. Non-sequential fractional ultrapulsed CO2 resurfacing of photoaged facial skin: preliminary clinical report. J Cosmet Laser Ther 2007;9:218-25. DOI PubMed

15. Bass LS, DelGuzzo M, Doherty S, Seckel B. Combined ablative and non-ablative fractional treatment for facial skin rejuvenation. Lasers Surg Med 2009;15:29. 